\title{
Microtomographic Study of Gabbro-Diabase Structural Transformations Under Compressive Loads
}

\author{
L. Vaisberg ${ }^{1(\bowtie)}$ and E. Kameneva ${ }^{2}$ \\ 1 «Mekhanobr-Tekhnika» REC, St. Petersburg, Russia \\ gornyi@mtspb.com \\ 2 Petrozavodsk State University, Petrozavodsk, Russia
}

\begin{abstract}
Transformations of the porous space structure for gabbro-diabase under compressive loads is studied. Quantitative assessment of respective structural parameters for the pore space is ensured through the application of $\mathrm{X}$-ray computer microtomography, enabling visualization of the internal threedimensional structure of each sample and a detailed quantitative analysis of the pore space structure for both separate sections and the entire sample volume. Differences in the number, sizes, shapes, connectivity and spatial distribution of pores are established. It is shown that, when the sample is destroyed, the structure of the pore space in its fragments is transformed as follows: intracrystalline pores are partially closing, with the simultaneous emergence of new pores of large capillary sizes, concentrated in the cracks. In terms of their structure, these cracks represent a system of interconnected pores containing micron-size mineral particles.
\end{abstract}

Keywords: Computer microtomography $\cdot$ Gabbro-diabase $\cdot$ Pore space

\section{Introduction}

In the current theory for the mechanism of disintegration of rocks, destruction is a process that develops in time. The formation and development of microscopic failures begin upon application of a loading force, either dynamic or static, and resume for the entire period the rock remains under load until fracture (Zhurkov 1980; Krivtsov 2007).

The existing works on rock destruction generally identify the most probable structural elements, along which the destruction processes tend to develop. These are pores, tiny fractures, intergrowth boundaries of mineral phases and intergrain boundaries. In this regard, porosity, understood as the sum of all cavities enclosed in the rock, including pores, pore channels, and tiny fractures, becomes a useful feature, linking the strength of a rock with the defects in its structure. The physical or total porosity of a rock, determined by calculation using known values of mineral and bulk densities, enables only indirectly assessing the transformations occurring in the rock microstructure during destruction. Unbiased data may only be obtained if the structure of the pore 
space is taken into account, including its dimensions, pore shapes, connectivity and spatial orientation (Romm 1985).

The purpose of this work is to study the transformations occurring in the microstructure of a rock during destruction. X-ray computer microtomography was selected as the method for the quantitative assessment of respective pore space structural parameters of rocks at the microscopic level.

\section{Methods and Approaches}

X-ray tomography (X-ray micro-CT) is a non-destructive method for studying the internal structure of solid materials, based on the dependence of the linear coefficient of $\mathrm{X}$-ray radiation attenuation on the chemical composition and density of the substance analyzed. Computer processing of shadow projections obtained by x-ray scanning of samples allows visualizing the internal three-dimensional structure of each sample and performing a detailed analysis of its morphometric and density characteristics both at separate sections and throughout the entire sample volume, obtaining quantitative values of respective parameters. The non-destructive nature of the method is an important advantage of X-ray microtomography, as it enables subsequent application of the same samples for other types of analysis, in particular, for establishing their strength characteristics.

A gabbro-diabase sample of cylindrical shape $(L=d)$ without visible defects was prepared for the studies. The gabbro-diabase sample was characterized by a massive texture and a uniform medium-grained structure. The size of mineral grains was up to 2-3 $\mathrm{mm}$.

The experiment included X-ray tomography of the gabbro-diabase sample with identification of the following parameters of the pore space: dimensions, shape, volume, specific surface, pore connectivity, spatial orientation, distribution, and pore density, both in separate tomographic Sections (2D system) and in the entire volume of the sample (3D system). The sample was then subjected to axial loading using a manual hydraulic press until fracture. The loading force was $192 \mathrm{MPa}$, the lateral pressure was $1 \mathrm{~atm}$, at $\mathrm{t}=20^{\circ} \mathrm{C}$, the loading time was $121 \mathrm{~s}$. The resulting fragment was then subjected to x-ray tomography.

The tomographic studies were carried out using SkyScan-1172 (Belgium) with resolutions of 0.5 to $27 \mu \mathrm{m}$. In the experiments, the samples were carefully oriented on the table with respect to the optical axis of the instrument; the tube was supplied with $100 \mathrm{~mA}$ and $100 \mathrm{kV}$; the X-ray power was $90 \mathrm{~W}$. The pixel size at the maximum magnification (nominal resolution) was $3.9 \mu \mathrm{m}$, which generally allows identifying pores of $4 \mu \mathrm{m}$ or larger. The table with the sample was rotated by $360^{\circ}$ in 0.25 increments. The subsequent reconstruction work was performed using Nrecon, CTan, and CTvol SkyScan software. 


\section{Results and Discussion}

The original sample contains crystals of $0.2-2.0 \mathrm{~mm}$, represented by plagioclase $(41.5 \%)$, actinolite $(47.0 \%)$, quartz $(3.6 \%)$, sphene $(5.7 \%)$ and biotite $(2.2 \%)$.

The porosity of the initial sample is $0.7 \%$. The pores in the sample volume are unevenly distributed. Plagioclase crystals have the highest porosity that is 4.1 to 4.9 times higher than the value for the entire sample (2.9-3.4\% vs $0.7 \%)$. In quartz, actinolite and biotite crystals, only individual pores are observed.

The concentration of pores in the original sample is $84.55 \mathrm{~mm}^{-3}$.

The largest pore size is $32-34 \mu \mathrm{m}$. In quantitative terms, pores of up to $10 \mu \mathrm{m}$ prevail. The largest pore size is $32-34 \mu \mathrm{m}$ with their median value of $5.6 \mu \mathrm{m}$. In quantitative terms, pores of up to $4-5 \mu \mathrm{m}(80.7 \%)$ prevail in this group.

In the fragment after fracture, the total pore concentration increases to $163.9 \mathrm{~mm}^{-3}$, super-capillary pores of 179 to $180 \mu \mathrm{m}$ are observed, and the porosity increases to $1.8 \%$ (Table 1).

Table 1. Results of microtomography (3D-system)

\begin{tabular}{l|l|l}
\hline \multirow{2}{*}{ Parameters } & \multicolumn{2}{|l}{ Values } \\
\cline { 2 - 3 } & Original sample & Fragment after sample fracture \\
\hline Pore fraction in sample volume, $\%$ & 0.7 & 1.8 \\
\hline Concentration of pores, $\mathrm{mm}^{-3}$ & 84.55 & 163.9 \\
\hline Largest pore size, $\mu \mathrm{m}$ & $32-34$ & $178-180$ \\
\hline Pore connectivity, $\%$ & 2.81 & 28.88 \\
\hline
\end{tabular}

Pore sphericity decreases from small pores to large pores. Pores of 4-5 $\mu \mathrm{m}$ are close to spherical in shape. Pores of 110-180 $\mu \mathrm{m}$ have elongated shapes, with their sphericity not exceeding 0.15 to 0.3 .

It can be seen on the tomographic sections (Fig. 1) that the fragments formed upon sample fracture contain incomplete cracks (not leading to the formation of fracture surfaces) with the length of $\mathrm{L}=7-8 \mathrm{~mm}$ and width of $\mathrm{h}=40-150 \mu \mathrm{m}$ (for main cracks) and of $\mathrm{h}=1-10 \mu \mathrm{m}$ (for feather cracks).

The study of the structure of the cracks shows that these are linear-plane sections consisting of interconnected cavities containing micron-size mineral particles (Fig. 2).

The availability of these cracks satisfactorily explains the increase in pore connectivity. In the original sample, the pore connectivity is $2.81 \%$; in the fractured fragment, it is an order of magnitude higher $(27.88 \%)$. The increase in connectivity indicates the association of small pores into larger pores. 


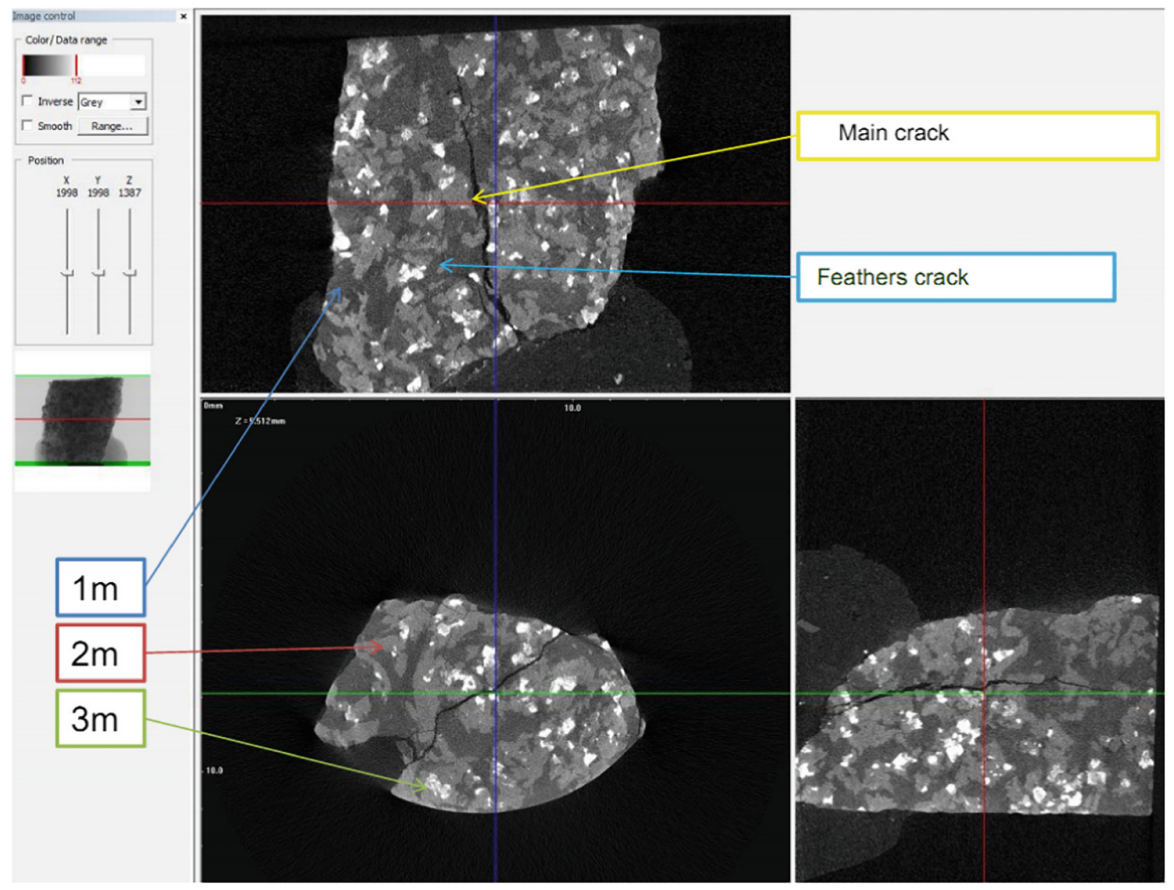

Fig. 1. Cracks in Gabbro-Diabase sample fragments (2D System) $1 \mathrm{~m}-$ Plagioclase; $2 \mathrm{~m}-$ Actinolite, 3-Sphene

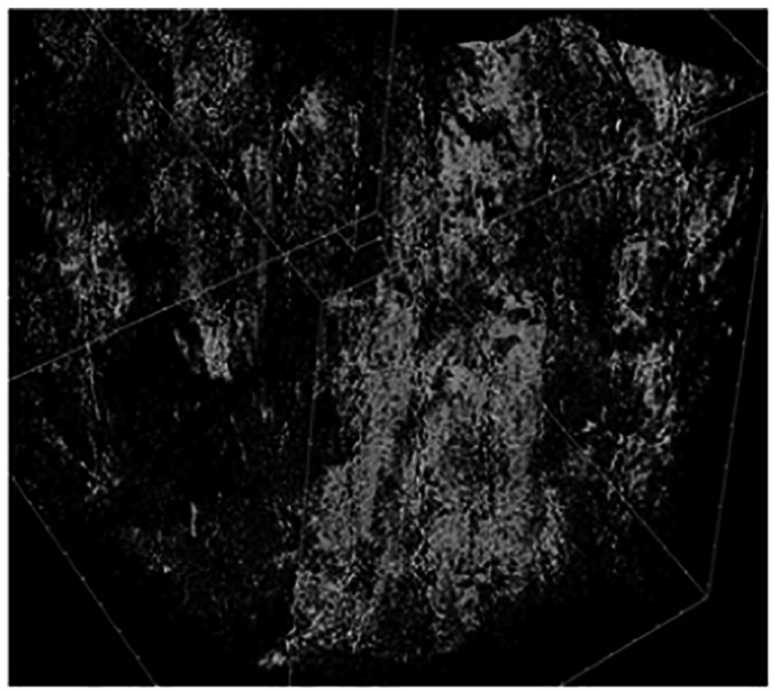

Fig. 2. Structure of cracks 
At the same time, a comparative tomographic study of the most porous mineral component, the plagioclase crystal, shows a decrease in pore concentration under compressive loads (Fig. 3). The plagioclase crystal porosity values are $3.23 \%$ for the original sample and $1.11 \%$ for the fractured fragment, which indicates that the intracrystalline pores are closing. Not only the finest pores, but also the larger ones are joined: the maximum pore size in the plagioclase crystal is $32 \mu \mathrm{m}$ in the original sample and $16 \mu \mathrm{m}$ in the fragment.

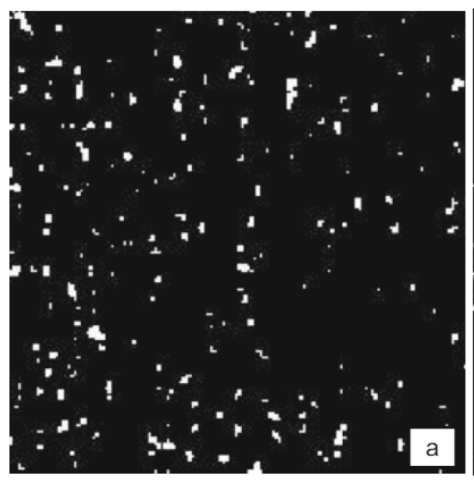

Porosity $-1,11 \%$

$\mathrm{D}_{\max }-16 \mathrm{um}$

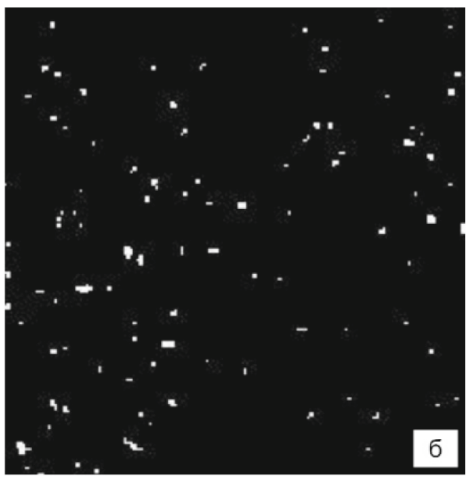

Porosity $-3,23 \%$

$\mathrm{D}_{\max }-32 \mathrm{um}$

Fig. 3. Pores (white) in Plagioclase crystal in original sample (a) and in its fractured fragment (b) in $2 \mathrm{D}$ system

The increase in the number of pores in the volume of the fragment formed after fracture, with the closing of intracrystalline pores, suggests that the newly formed pores are mainly concentrated in the cracks. In terms of their structures, these cracks represent a system of interconnected pores containing micron-size mineral particles.

\section{Conclusions}

When a gabbro-diabase sample is fractured under the action of a compressive load, the structure of the pore space is transformed in the fragments formed as follows: intracrystalline pores are partially closing, with the simultaneous emergence of new pores of large capillary sizes, concentrated in the cracks.

Acknowledgements. The study was supported by the grant of the Russian Science Foundation (project No. 17-79-30056). 


\section{References}

Krivtsov AM (2007) Deformation and fracture of solids with microstructure, Fizmatlit, Moscow Romm YeS (1985) Structural models of pore space of rocks, Nedra, Leningrad

Zhurkov SN (1980) On the question of the physical nature of the strength. J Solid State Phys 22 (11):13-15

Open Access This chapter is licensed under the terms of the Creative Commons Attribution 4.0 International License (http://creativecommons.org/licenses/by/4.0/), which permits use, sharing, adaptation, distribution and reproduction in any medium or format, as long as you give appropriate credit to the original author(s) and the source, provide a link to the Creative Commons license and indicate if changes were made.

The images or other third party material in this chapter are included in the chapter's Creative Commons license, unless indicated otherwise in a credit line to the material. If material is not included in the chapter's Creative Commons license and your intended use is not permitted by statutory regulation or exceeds the permitted use, you will need to obtain permission directly from the copyright holder.

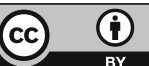

